\title{
Biobanks and future emerging technologies: new approaches, new pre- analytical challenges
}

\section{Eva Ortega-Paino $^{1 凶}$, Tomas Klingström² ${ }^{2}$, Johanna Ekström ${ }^{1}$}

'BBMRI.se Service Center for Southern Sweden, Medicon Village (406), Lund University, Lund, Sweden ${ }^{2} S L U$ Global Bioinformatics Centre, Uppsala University, Uppsala, Sweden

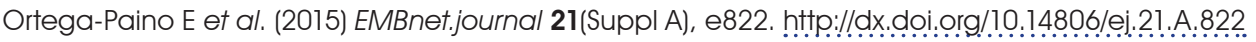

Establishing a Biobank is a major long-term commitment and samples collected today must be relevant for next generation techniques far into the future.

It is therefore crucial for biobanks to be "future compatible" and rely on sampling and storage methods to maximize the future value of the collections. In it's most basic form such future compatibility may be limited to careful sample management such as a strict adherence to quality management and best practices as laid down by authoritative sources such as ISBER (International Society for Biological and Environmental Repositories, 2012) guidelines and BRISQ (Moore et al., 2011; 2012; 2013), among Others.

This approach will be sufficient, if adhered to, for most current generation sequencing techniques where read-lengths are limited to 150 $500 \mathrm{bp}$ and if biobank samples intended for sequencing carry an abundance of RNA or DNA. But single cell sequencing of circulating tumor cells, DNA methylation analysis and proteogenomic studies, where DNA, RNA and protein from the same sample is analysed (Nesvizhskii, 2014), require new standards for sample collection and storage.

Therefore, and looking at the future, it would be very desirable to run studies similar to SPIDIARNA (Malentacchi et al., 2014) and SPIDIA-DNA (Malentacchi, 2013) to ensure that samples can be used for proteogenomics and other applica- tions where proteomics and nucleic acid based assays are combined. But to achieve this it is necessary to create biobank cohorts with sufficient collection, quality and storage conditions.

\section{Acknowledgements}

Funding for this work has been provided by BBMRI.se.

\section{References}

International Society for Biological and Environmental Repositories (2012) 2012 Best Practices for Repositories: Collection, Storage, Retrieval, and Distribution of Biological Materials for Research. Biopreservation and Biobanking 10(2), 81-161. http://dx.doi.org/10.1089/bio. 2012.1022

Malentacchi F, Pazzagli M, Simi L, Orlando C. et al. (2014) SPIDIA-RNA: second external quality assessment for the pre-analytical phase of blood samples used for RNA based analyses. PLoS One 9(11):e112293. http://dx.doi. org/10.1371/journal.pone.0112293.

Malentacchi F, Pazzagli M, Simi L, Orlando C, Wyrich R et al. (2013) SPIDIA-DNA: an External Quality Assessment for the pre-analytical phase of blood samples used for DNAbased analyses. Clin Chim Acta. 424, 274-286. htțp:/l! dx.doi.org/10.1016/j.cca. 2013.05.012

Moore HM, Kelly A, Jewel SD, McShane LM et al. (2011) Biospecimen reporting for improved study quality (BRISQ). J Proteome Res. 10(8), 3429-38, ntttp://dx.doi.org/10.1021/! pr200021n

Moore HM, Kelly A, McShane LM, VaughtJ (2012) Biospecimen reporting for improved study quality (BRISQ). Clin Chim

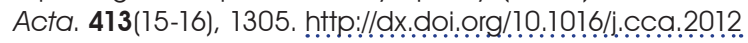
.04 .013

Moore HM, KellyA, McShane LM, VaughtJ (2013) Biospecimen reporting for improved study quality (BRISQ). Transfusion 53(7):el. http:///dx.doi.org/10.1!11/trtu.12281

Nesvizhskii Al (2014) Proteogenomics: concepts, applications and computational strategies. Nature Methods 11(11), 1114-1125. http:///dx.doi.org/10.1038/nmeth.3144 